Proceedings of the Edinburgh Mathematical Society (2007) 50, 343-362 (C)

DOI:10.1017/S0013091504000902 Printed in the United Kingdom

\title{
PRODUCTS OF IDEMPOTENT ENDOMORPHISMS OF RELATIVELY FREE ALGEBRAS WITH WEAK EXCHANGE PROPERTIES
}

\author{
JOHN FOUNTAIN AND VICTORIA GOULD \\ Department of Mathematics, University of York, Heslington, \\ York YO10 5DD, UK (jbf1@york.ac.uk; varg1@york.ac.uk)
}

(Received 20 September 2004)

\begin{abstract}
If $A$ is a stable basis algebra of rank $n$, then the set $S_{n-1}$ of endomorphisms of rank at most $n-1$ is a subsemigroup of the endomorphism monoid of $A$. This paper gives a number of necessary and sufficient conditions for $S_{n-1}$ to be generated by idempotents. These conditions are satisfied by finitely generated free modules over Euclidean domains and by free left $T$-sets of finite rank, where $T$ is cancellative monoid in which every finitely generated left ideal is principal.
\end{abstract}

Keywords: basis; exchange property; endomorphism monoid; idempotents

2000 Mathematics subject classification: Primary 20M20; 08A35

Secondary 20M10; 08A05

\section{Introduction}

One of the most natural ways in which semigroups arise is as the semigroup of endomorphisms End $A$ of a given (universal) algebra $A$. For certain $A$ the structure of End $A$ is well known. Our motivating examples are the cases where $A$ is a finite set (an algebra with no operations) or a finite-dimensional vector space. In each case, End $A$ is the union of a finite chain of ideals and the Rees quotients of successive ideals are completely 0 -simple.

In the abstract theory of semigroups, idempotents play a major role in structural investigations. It is therefore natural to attempt to describe the subsemigroup $\langle E\rangle$ generated by the idempotents $E$. In particular, what is $\langle E\rangle$, where $E$ is the set of idempotents of End $A$ for some algebra $A$ ? To make results easier to state we exclude the identity map from $E$ (hence from $\langle E\rangle$ ). Howie [14] showed that if $A$ is a finite set, then $\langle E\rangle$ is the semigroup of non-bijective mappings; the analogous statement for finite-dimensional vector spaces over a field was proved by Erdős [5]. An alternative proof of the latter was given by Dawlings [4], and the result was generalized to finite-dimensional vector spaces over division rings by Laffey [16].

More recently, Fountain and Lewin [10] showed that the results of Howie, Erdös, Dawlings and Laffey are special cases of a more general theorem for independence algebras. 
Independence algebras (also known as $v^{*}$-algebras) are defined by two conditions: the first says that the closure operator $\langle\cdot\rangle$ satisfies the exchange property; the second requires that independent sets be free generators. Sets and vector spaces are examples of independence algebras. Any subset and, in particular, any subalgebra of an independence algebra $A$ has a well-defined rank, namely, the cardinal of any maximal independent subset it contains. We define the rank of $\alpha \in$ End $A$ to be the rank of $\operatorname{Im} \alpha$. If $A$ has finite rank $n$, then $A$ is the union of a finite chain of ideals with completely 0-simple Rees quotients [11], and $\langle E\rangle$ consists of all endomorphisms of rank strictly less than $n[\mathbf{1 0}]$.

The endomorphism monoid of an independence algebra is regular. But, perhaps surprisingly, regularity of End $A$ is not necessary for the above results concerning $\langle E\rangle$. For example, Laffey [16] showed that if $A$ is a free module of finite rank $n$ over a Euclidean domain, then $\langle E\rangle$ is the subsemigroup of endomorphisms of rank strictly less than $n$.

Basis algebras provide a class of algebras in which we attempt to put the results of Laffey, and later work of Fountain [7] and Ruitenberg [18], into a more general setting. In a basis algebra the closure operator PC (pure closure) satisfies the exchange property and directly independent subsets (that is, subsets independent with respect to PC) are free generators. Finitely generated free left modules over Bezout domains and free left $T$-sets over cancellative left Ore monoids are examples of basis algebras. As in the case of independence algebras, rank is well defined for subsets, subalgebras and endomorphisms of basis algebras, where now the rank of a subset is the cardinality of a maximal directly independent set that it contains. In an earlier paper [9] we showed that if $A$ is a basis algebra of finite rank of a special kind that we call stable, then End $A$ is the union of a finite chain of ideals with Rees quotients that are primitive abundant semigroups in which all the non-zero idempotents are $\mathcal{D}$-related. The latter have a structure analogous to that of completely 0 -simple semigroups, being isomorphic to a Rees matrix semigroup over a cancellative monoid [6]. This structure allows us to give a number of equivalent sufficient conditions for $\langle E\rangle$ to consist of all endomorphisms of rank strictly less than $n$. We conclude the paper with a number of examples of basis algebras satisfying these conditions.

The structure of the paper is as follows. In $\S 1$ we give a brief résumé of the concepts and definitions behind basis algebras. Section 2 gives some facts concerning endomorphisms of basis algebras together with technical results needed for our result on products of idempotents. Section 3 is devoted to proving the latter. In $\S 4$ we illustrate the result with a number of examples.

Further details of the results we quote in $\S \S 1$ and 2 may be found in the two predecessors of this article $[\mathbf{8}, \mathbf{9}]$.

\section{Basis algebras}

By an algebra $A$ we mean an algebra in the sense of universal algebra. For basic ideas of universal algebra we refer the reader to $[\mathbf{2}],[\mathbf{1 2}]$ or $[\mathbf{1 7}]$. For convenience the symbols $m$ and $n$ are always reserved for natural numbers.

A constant in an algebra $A$ is the image of a basic nullary operation. If $A$ has any constants, then $\langle\emptyset\rangle$ denotes the subalgebra generated by them, and $\langle\emptyset\rangle$ consists of those 
elements $a$ for which there is a unary term operation with unique value $a$ (see, for example, [12, p. 40, Corollary 3]). If an algebra has no constants, then we make the convention that $\emptyset$ is a subalgebra so that in this case, $\langle\emptyset\rangle=\emptyset$. We say that an algebra $A$ is constant if $A=\langle\emptyset\rangle$. All the algebras studied in this paper have the property that if the algebra has no constants and more than one element, then none of the unary term operations are constant functions.

We are concerned with algebras having two closure operators. One is the standard subalgebra closure operator $\langle\cdot\rangle$, the other is the operator PC.

For an element $a$ of an algebra $A$ and a subset $X$ of $A$, we write $a \prec X$ if

$$
a \in\langle\emptyset\rangle \quad \text { or } \quad\langle a\rangle \cap\langle X\rangle \neq\langle\emptyset\rangle
$$

and we put

$$
\mathrm{PC}(X)=\{a \in A: a \prec X\} .
$$

The operator PC need not be a closure operator. Where it is, it is algebraic and the closed subsets are subalgebras. We say that $A$ is a weak exchange algebra if $\mathrm{PC}$ is a closure operator satisfying the exchange property. The latter ensures that subsets of $A$ have well-defined rank, where the rank of $X$ is the cardinality of any maximum PCindependent subset of $X$. We refer to PC-independent subsets as directly independent. Clearly, rank is monotonic. We require the following result from [8], most of which is classical and can be taken from [2].

Lemma 1.1 (Fountain and Gould [8]). Let $X$ be a subset of a weak exchange algebra $A$. Then

(i) $\operatorname{rank}\langle X\rangle=\operatorname{rank} X=\operatorname{rank} \operatorname{PC}(X) \leqslant|X|$;

(ii) if $X$ is finite and $\operatorname{rank}\langle X\rangle=|X|$, then $X$ is directly independent.

A subset $X$ of an algebra $A$ is $A$-free if every function from $X$ to $A$ can be extended to a morphism from $\langle X\rangle$ to $A$. An $A$-free subset $X$ of $A$ is a basis of $A$ if $X \cap\langle\emptyset\rangle=\emptyset$ and $X$ generates $A$. A weak independence algebra $A$ is a weak exchange algebra in which every directly independent set is $A$-free.

We say that an algebra $A$ is torsion free if each unary term operation $t$, such that $\operatorname{Im} t$ is not contained in the constant subalgebra, is injective. Notice that any constant algebra is therefore torsion free. It follows from the results of [8] that a basis of a torsion-free weak independence algebra is exactly the same thing as a generating set that is directly independent.

In a weak independence algebra $A$ a pure subalgebra is a subalgebra that is PC-closed. We remark that from Lemma 4.2 of [8], any subalgebra of $A$ is a weak independence algebra. From Corollary 1.12 of [8], if $B$ and $C$ are subalgebras with $B \subseteq C, B$ pure in $C$ and $C$ pure in $A$, then $B$ is pure in $A$.

We are interested in weak independence algebras in which pure subalgebras have bases which can be extended to bases of the parent algebra.

Lemma 1.2 (Fountain and Gould [8]). Let $A$ be a torsion free weak independence algebra. If $X$ is a basis for $A$ and $Y \subseteq X$, then $\langle Y\rangle$ is pure. 
A basis algebra $A$ is a torsion-free weak independence algebra which satisfies the following condition:

if $P, Q$ are pure subalgebras in $A$ with $P \subseteq Q$, and $X$ is

a basis for $P$, then there is a basis $Y$ for $Q$ with $X \subseteq Y$.

It follows from this definition that every pure subalgebra of a basis algebra has a basis, and, in particular, every basis algebra has a basis. Moreover, from Proposition 7.2 of [8], every pure subalgebra of a basis algebra is itself a basis algebra.

Suppose now that $A$ is a basis algebra, $\operatorname{rank} A=n$ and $B$ is a pure subalgebra of $A$ with $\operatorname{rank} B=n$. Since any basis of $B$ can be extended to a basis of $A$, we must have that $A=B$.

Finally, we say that a basis algebra $A$ is stable if every subalgebra of $A$ having a generating set of cardinality at most rank $A$ has a basis.

The following result is a consequence of the monotonicity of rank.

Lemma 1.3. Let $A$ be a stable basis algebra and let $B$ be a pure subalgebra of $A$. Then $B$ is a stable basis algebra.

Independence algebras are stable basis algebras. If $R$ is a Bezout domain (that is, a domain in which every finitely generated one-sided ideal is principal), then a free left $R$-module of finite rank is a stable basis algebra [8]. Our third canonical example of a stable basis algebra is a free left $T$-act over a cancellative principal left ideal monoid $T$. Further details of these examples may be found in $[\mathbf{8}]$ and in the final section of this paper.

\section{Endomorphisms of basis algebras}

This section gives some necessary background material on the monoid of endomorphisms of a stable basis algebra. We assume that the reader is familiar with elementary semigroup theory, including Green's relations $\mathcal{R}, \mathcal{L}, \mathcal{H}$ and $\mathcal{D}$.

On a semigroup $S$, the relation $\mathcal{R}^{*}$ is defined by the rule that for any $a, b \in S, a \mathcal{R}^{*} b$ if and only if $a \mathcal{R} b$ in some oversemigroup of $S$. Equivalently, $a \mathcal{R}^{*} b$ if and only if, for all $x, y \in S^{1}$,

$$
x a=y a \quad \text { if and only if } x b=y b .
$$

It is easy to see that if $a, b$ are regular elements, then $a \mathcal{R} b$ if and only if $a \mathcal{R}^{*} b$. In general, however, the relation $\mathcal{R}$ is strictly contained in $\mathcal{R}^{*}$. The relation $\mathcal{L}^{*}$ is defined dually; we put $\mathcal{D}^{*}=\mathcal{L}^{*} \vee \mathcal{R}^{*}$.

Lemma 2.1. Let $x, y$ be elements of a semigroup $S$ such that $x y$ is regular. If $x y \mathcal{R}^{*} x$, then $x$ is regular. Dually, if $x y \mathcal{L}^{*} y$, then $y$ is regular.

Proof. Since $x y$ is regular, $x y=(x y) t(x y)$ for some $t \in S$. Suppose now that $x y \mathcal{R}^{*} x$. Then, since $1 x y=(x y t) x y$, we have $1 x=(x y t) x$ so that $x$ is regular.

For the convenience of the reader we now give a number of results from $[\mathbf{9}]$. 
Lemma 2.2 (Fountain and Gould [9, Corollary 4.2]). Let $A$ be a basis algebra. Then, for any $\alpha, \beta \in$ End $A$,

$$
\alpha \mathcal{R}^{*} \beta \quad \text { if and only if } \operatorname{Ker} \alpha=\operatorname{Ker} \beta .
$$

Lemma 2.3 (Fountain and Gould [9, Corollary 4.6]). Let $A$ be a basis algebra. Then, for any $\alpha, \beta \in$ End $A$,

$$
\alpha \mathcal{L}^{*} \beta \quad \text { if and only if } \mathrm{PC}(\operatorname{Im} \alpha)=\mathrm{PC}(\operatorname{Im} \beta) .
$$

Lemma 2.4 (Fountain and Gould [9, Lemma 4.7]). Let $\alpha \in$ End $A$, where $A$ is a basis algebra. Then $\alpha$ is regular if and only if $\operatorname{Im} \alpha=\mathrm{PC}(\operatorname{Im} \alpha)$.

Lemma 2.5 (Fountain and Gould [9, Theorem 4.9]). Let $A$ be a stable basis algebra. Then every $\mathcal{H}^{*}$-class of End $A$ contains a regular element. Consequently, $\mathcal{D}^{*}=$ $\mathcal{R}^{*} \circ \mathcal{L}^{*}=\mathcal{L}^{*} \circ \mathcal{R}^{*}$.

We briefly consider certain ideals in End $A$ where $A$ is a stable basis algebra. First, for $\alpha \in \operatorname{End} A$, we define $\operatorname{rank} \alpha=\operatorname{rank} \operatorname{Im} \alpha$. From [9], we have the following easy but crucial result.

Lemma 2.6 (Fountain and Gould [9, Lemma 6.1]). If $\alpha, \beta$ are endomorphisms of a stable basis algebra $A$, then $\operatorname{rank} \alpha \beta \leqslant \min \{\operatorname{rank} \alpha, \operatorname{rank} \beta\}$.

Now for a non-negative integer $m$ with $m \leqslant \operatorname{rank} A$ we define

$$
S_{m}=\{\alpha \in \text { End } A: \operatorname{rank} \alpha \leqslant m\} .
$$

It follows from Lemma 2.6 that $S_{m}$ is an ideal of End $A$.

Lemma 2.7 (Fountain and Gould [9, Lemma 7.2]). Let $A$ be a basis algebra. Then $S_{0} \neq \emptyset$ if and only if $\langle\emptyset\rangle \neq \emptyset$. When $S_{0} \neq \emptyset$, it is a left zero semigroup.

Lemma 2.8 (Fountain and Gould [9, Proposition 7.3]). For a positive integer $m$ with $m \leqslant \operatorname{rank} A$, the Rees quotient semigroup $S_{m} / S_{m-1}$ is a primitive abundant semigroup in which all the non-zero idempotents are $\mathcal{D}$-related. Consequently, $S_{m} / S_{m-1}$ is isomorphic to a Rees matrix semigroup $\mathcal{M}^{0}(S ; I, \Lambda ; P)$ where $S$ is a cancellative monoid, and each row and column of $P$ contains a unit of $S$.

In the above result, the set $I$ indexes the $\mathcal{R}^{*}$-classes of $\mathcal{M}^{0}$ and the set $\Lambda$ the $\mathcal{L}^{*}$-classes. Together with Lemma 2.5 of [9] we deduce the following.

Corollary 2.9. Let $A$ be a stable basis algebra. Then, for any $\alpha, \beta \in$ End $A$, if

$$
\operatorname{rank} \alpha=\operatorname{rank} \beta=\operatorname{rank} \alpha \beta=n,
$$

we have

$$
\alpha \mathcal{R}^{*} \alpha \beta \mathcal{L}^{*} \beta
$$


By an endomorphism pair of a stable basis algebra $A$, we mean a pair $(\rho, B)$ where $B$ is a pure subalgebra of $A$ and $\rho$ is a congruence on $A$ such that $A / \rho$ is isomorphic to $B$. For such an endomorphism pair, we put

$$
H_{(\rho, B)}^{*}=\{\alpha \in \text { End } A: \operatorname{Ker} \alpha=\rho \text { and } \operatorname{PC}(\operatorname{Im} \alpha)=B\} .
$$

The following is a consequence of Lemmas 2.2 and 2.3 above.

Proposition 2.10 (Fountain and Gould [9, Lemma 4.10]). Let $A$ be a stable basis algebra. Then the $\mathcal{H}^{*}$-classes of End $A$ are precisely the subsets of the form $H_{(\rho, B)}^{*}$ where $(\rho, B)$ is an endomorphism pair.

For an algebra $A$ and a subset $H$ of $A \times A$ we denote by $\langle\langle H\rangle$ the smallest congruence on $A$ containing $H$. If $A$ is a stable basis algebra, we say that an endomorphism pair is idempotent if the corresponding $\mathcal{H}^{*}$-class contains an idempotent.

Proposition 2.11. Let $A$ be a stable basis algebra of rank $n$ where $n \geqslant 2$. Suppose that $\left\{x_{1}, \ldots, x_{r}, y_{1}, \ldots, y_{n-r}\right\}$ is a basis for $A$ where $1 \leqslant r \leqslant n-1$. Then

$$
(\rho, B)=\left(\left\langle\left(x_{1}, y_{1}\right), \ldots,\left(x_{1}, y_{n-r}\right)\right\rangle,\left\langle x_{1}, \ldots, x_{r}\right\rangle\right)
$$

is an idempotent endomorphism pair.

Proof. Define $\alpha \in$ End $A$ by

$$
\begin{aligned}
x_{i} \alpha=x_{i}, & 1 \leqslant i \leqslant r, \\
y_{j}=x_{1}, & 1 \leqslant j \leqslant n-r .
\end{aligned}
$$

Clearly, $\alpha=\alpha^{2}$ and $\operatorname{Im} \alpha=\left\langle x_{1}, \ldots, x_{r}\right\rangle$.

Certainly $\rho \subseteq$ Ker $\alpha$. To show the opposite inclusion, suppose that $s, t$ are terms and

$$
s\left(x_{1}, \ldots, x_{r}, y_{1}, \ldots, y_{n-r}\right) \alpha=t\left(x_{1}, \ldots, x_{r}, y_{1}, \ldots, y_{n-r}\right) \alpha,
$$

so that

$$
s\left(x_{1}, \ldots, x_{r}, x_{1}, \ldots, x_{1}\right)=t\left(x_{1}, \ldots, x_{r}, x_{1}, \ldots, x_{1}\right) .
$$

We have

$$
\begin{aligned}
s\left(x_{1}, \ldots, x_{r}, y_{1}, \ldots, y_{n-r}\right) & \rho s\left(x_{1}, \ldots, x_{r}, x_{1}, \ldots, x_{1}\right) \\
= & t\left(x_{1}, \ldots, x_{r}, x_{1}, \ldots, x_{1}\right) \rho t\left(x_{1}, \ldots, x_{r}, y_{1}, \ldots, y_{n-r}\right)
\end{aligned}
$$

so that Ker $\alpha \subseteq \rho$ as required. The result now follows from Lemma 2.4 and Proposition 2.10 .

For a basis algebra $A$, any subset has a well-defined rank. Not every subalgebra need have a basis, that is, a maximal directly independent generating set. However, for stable basis algebras, every image of a morphism has a basis. This is part of the content of the following technical result. 
Lemma 2.12. Let $A$ be a stable basis algebra of finite rank $n$ and let $\alpha \in$ End $A$ with $\operatorname{rank} \alpha=m$. Then there is a basis $\left\{x_{1}, \ldots, x_{n}\right\}$ of $A$ such that $\operatorname{Im} \alpha$ has basis $\left\{x_{1} \alpha, \ldots, x_{m} \alpha\right\}$.

Proof. That $\operatorname{Im} \alpha$ has a basis is Lemma 4.8 of [9]. Suppose that $\left\{z_{1} \alpha, \ldots, z_{m} \alpha\right\}$ is a basis of $\operatorname{Im} \alpha$. By Proposition 4.2 of $[8], Z=\left\{z_{1}, \ldots, z_{m}\right\}$ is directly independent. Now $\operatorname{rank}\langle Z\rangle=\operatorname{rank} \mathrm{PC}(Z)=m$, so that $\mathrm{PC}(Z)=\left\langle x_{1}, \ldots, x_{m}\right\rangle$ for some directly independent set $\left\{x_{1}, \ldots, x_{m}\right\}$ where $\left\{x_{1}, \ldots, x_{n}\right\}$ is a basis for $A$. We have

$$
\begin{aligned}
\operatorname{Im} \alpha & =\left\langle z_{1} \alpha, \ldots, z_{m} \alpha\right\rangle \\
& =\langle Z\rangle \alpha \subseteq \operatorname{PC}(Z) \alpha \\
& =\left\langle x_{1}, \ldots, x_{m}\right\rangle \alpha \\
& =\left\langle x_{1} \alpha, \ldots, x_{m} \alpha\right\rangle \subseteq \operatorname{Im} \alpha
\end{aligned}
$$

so that $\operatorname{Im} \alpha=\left\langle x_{1} \alpha, \ldots, x_{m} \alpha\right\rangle$. By Lemma $1.1,\left\{x_{1} \alpha, \ldots, x_{m} \alpha\right\}$ is directly independent and so it is a basis of $\operatorname{Im} \alpha$.

We end this section with a proposition essentially taken from $[\mathbf{6}]$, which appears explicitly in [7]. This result is crucial in simplifying our arguments in the next section.

Proposition 2.13 (Fountain [6]). Let $S$ be a semigroup in which every $\mathcal{H}^{*}$-class contains a regular element. Let $e$ be an idempotent of $S$ and let $a$ be an element of the $\mathcal{D}^{*}$-class of $e$. Let $c, d$ be regular elements in the $\mathcal{H}^{*}$-classes $L_{e}^{*} \cap R_{a}^{*}$ and $R_{e}^{*} \cap L_{a}^{*}$. Then the function $\theta: H_{e}^{*} \rightarrow H_{a}^{*}$ given by $b \theta=c b d$ is a bijection.

\section{The criteria}

An immediate consequence of Lemma 2.4 is that for any idempotent $\varepsilon \in$ End $A$, where $A$ is a stable basis algebra, we have that $\operatorname{Im} \varepsilon=\mathrm{PC}(\operatorname{Im} \varepsilon)$. It follows that if $\operatorname{rank} \varepsilon=\operatorname{rank} A$ is finite, then $\varepsilon=I_{A}$. With this in mind, and to fit in with established terminology, for a stable basis algebra $A$ of finite rank $n$ we put

$$
E^{A}=\left\{\varepsilon=\varepsilon^{2} \in \text { End } A: \operatorname{rank} \varepsilon<n\right\},
$$

and, for $1 \leqslant r \leqslant n-1$,

$$
E_{n-r}^{A}=\left\{\varepsilon=\varepsilon^{2} \in \operatorname{End} A: \operatorname{rank} \varepsilon=r\right\} .
$$

The aim of this section is to give a number of sufficient conditions on a stable basis algebra $A$ of rank $n$ for the non-identity idempotents to generate $S_{n-1}$, that is, for $\left\langle E^{A}\right\rangle=S_{n-1}$. Notice that by Lemma $2.8,\left\langle E^{A}\right\rangle$ is a subsemigroup of $S_{n-1}$. If $\operatorname{rank} A=0$, then End $A$ is trivial. If $\operatorname{rank} A=1$, then by Lemma $2.7, S_{0}$ is either empty or is a left zero semigroup, in which case $E^{A}=S_{0}$ and certainly $\left\langle E^{A}\right\rangle=S_{0}$. We concentrate therefore on the case where $n \geqslant 2$. The conditions we give on $A$ are hereditary conditions, that is, they must be satisfied by all pure subalgebras of $A$ of rank at least 2. Recall that if $B$ is a pure subalgebra of $A$, then $\operatorname{rank} B \leqslant \operatorname{rank} A$, and that if $\operatorname{rank} B=\operatorname{rank} A$, then $A=B$. Several algebras are involved in the ensuing discussion, and so, for clarity, we write $S_{r}^{B}$ for the ideal of endomorphisms of rank no greater than $r$ in End $B$. 
Theorem 3.1. Let $A$ be a stable basis algebra of rank $n$. Then the following conditions are equivalent.

(1) $\left\langle E^{B}\right\rangle=S_{m-1}^{B}$ for all integers $m$ with $2 \leqslant m \leqslant n$ and all pure subalgebras $B$ of $A$ with $\operatorname{rank} B=m$.

(2) $\left\langle E_{1}^{B}\right\rangle=S_{m-1}^{B}$ for all integers $m$ with $2 \leqslant m \leqslant n$ and all pure subalgebras $B$ of $A$ with $\operatorname{rank} B=m$.

(3) $\left\langle E_{m-r}^{B}\right\rangle=S_{r}^{B}$ for $1 \leqslant r \leqslant m-1$ for all integers $m$ with $2 \leqslant m \leqslant n$ and all pure subalgebras $B$ of $A$ with $\operatorname{rank} B=m$.

(4) For all integers $m$ with $2 \leqslant m \leqslant n$ and every pure subalgebra $B$ of $A$ with $\operatorname{rank} B=$ $m$, every $\mathcal{H}^{*}$-class of End $B$ contained in $D_{m-1}^{*}$ contains a regular element that is a product of idempotents (of rank $m-1$ ).

(5) For every pure subalgebra $B$ of $A$ with $\operatorname{rank} B \geqslant 2$ and for any endomorphism pair $(\rho, C)$ of $B$, where $\operatorname{rank} C=\operatorname{rank} B-1$, there are a finite number of pure subalgebras $C_{1}, \ldots, C_{k}$ of $B$ and a finite number of congruences $\rho_{1}, \ldots, \rho_{k}$ on $B$ such that $C=C_{1}, \rho=\rho_{k}$ and, for all $i, j$ with $1 \leqslant i \leqslant k, 1 \leqslant j \leqslant k-1,\left(\rho_{i}, C_{i}\right)$ and $\left(\rho_{j}, C_{j+1}\right)$ are idempotent endomorphism pairs.

Proof. Let $B$ be any pure subalgebra of $A$. Since $A$ is stable, it follows from Lemma 1.3 that $B$ is a stable basis algebra and we can therefore call upon the results of $\S 2$ for End $B$.

That (3) implies (2) is clear. That (2) implies (1) is a consequence of $S_{m-1}^{B}$ being an ideal of End $B$. That (1) implies (2) is an easy consequence of the next lemma.

Lemma 3.2. Let $B$ be a stable basis algebra of rank $m$ with $m \geqslant 2$ and let $\alpha \in$ End $B$ with $0 \leqslant \operatorname{rank} \alpha=r \leqslant m-2$. Then there exist $\beta, \gamma \in \operatorname{End} B$ with $\operatorname{rank} \beta=\operatorname{rank} \gamma=r+1$ such that $\alpha=\beta \gamma$ and $\gamma$ is idempotent.

Proof. If $\operatorname{rank} \alpha=0$ so that $\operatorname{Im} \alpha=\langle\emptyset\rangle$, let $\left\{x_{1}, \ldots, x_{m}\right\}$ be a basis for $B$ and define $\beta, \gamma \in$ End $B$ by

$$
\begin{aligned}
& x_{i} \beta= \begin{cases}x_{m} & \text { if } i=m, \\
x_{i} \alpha & \text { otherwise, }\end{cases} \\
& x_{i} \gamma= \begin{cases}x_{1} & \text { if } i=1, \\
x_{i} \alpha & \text { otherwise. }\end{cases}
\end{aligned}
$$

Since $2 \leqslant m$, it is clear that $\alpha=\beta \gamma$, that $\beta, \gamma$ have rank 1 and, in this case, that both $\beta$ and $\gamma$ are idempotent.

If $\operatorname{rank} \alpha=r>0$, then by Lemma 2.12 , there is a basis $\left\{x_{1}, \ldots, x_{m}\right\}$ for $B$ such that $\left\{x_{1} \alpha, \ldots, x_{r} \alpha\right\}$ is a basis for $\operatorname{Im} \alpha$. Let $\left\{y_{1}, \ldots, y_{r}\right\}$ be a basis for $\mathrm{PC}(\operatorname{Im} \alpha)$, where $\left\{y_{1}, \ldots, y_{m}\right\}$ is a basis for $B$. Define $\beta \in$ End $B$ by

$$
x_{i} \beta= \begin{cases}x_{i} \alpha & \text { if } 1 \leqslant i \leqslant r \\ y_{r+1} & \text { if } i=r+1\end{cases}
$$


Then

$$
\operatorname{Im} \beta=\left\langle x_{1} \alpha, \ldots, x_{r} \alpha, y_{r+1}, x_{r+2} \alpha, \ldots, x_{n} \alpha\right\rangle=\left\langle x_{1} \alpha, \ldots, x_{r} \alpha, y_{r+1}\right\rangle
$$

so that by Lemma 1.1, $\operatorname{rank} \beta \leqslant r+1$. But $\operatorname{Im} \alpha \subseteq \operatorname{Im} \beta$ so that $\mathrm{PC}(\operatorname{Im} \alpha) \subseteq \mathrm{PC}(\operatorname{Im} \beta)$. Hence

$$
\left\{y_{1}, \ldots, y_{r}, y_{r+1}\right\} \subseteq \mathrm{PC}(\operatorname{Im} \beta)
$$

so that

$$
r+1 \leqslant \operatorname{rank} P C(\operatorname{Im} \beta)=\operatorname{rank}(\operatorname{Im} \beta)=\operatorname{rank} \beta,
$$

giving $\operatorname{rank} \beta=r+1$.

We now define $\gamma \in$ End $B$ by putting

$$
y_{i} \gamma= \begin{cases}y_{i} & \text { if } 1 \leqslant i \leqslant r, \\ x_{r+1} \alpha & \text { if } i=r+1, \\ y_{r+2} & \text { if } r+2 \leqslant i \leqslant m .\end{cases}
$$

Then

$$
\operatorname{Im} \gamma=\left\langle y_{1}, \ldots, y_{r}, x_{r+1} \alpha, y_{r+2}\right\rangle=\left\langle y_{1}, \ldots, y_{r}, y_{r+2}\right\rangle
$$

so that $\operatorname{rank} \gamma=r+1$.

We have

$$
x_{r+1} \beta \gamma=y_{r+1} \gamma=x_{r+1} \alpha
$$

and, for $i \neq r+1$,

$$
x_{i} \beta \gamma=x_{i} \alpha \gamma=x_{i} \alpha,
$$

since $a \gamma=a$ for all $a \in \operatorname{PC}(\operatorname{Im} \alpha)$. Thus $\alpha=\beta \gamma$. Since $\operatorname{Im} \gamma=\left\langle y_{1}, \ldots, y_{r}, y_{r+2}\right\rangle$, we see that $\gamma$ is idempotent.

Now let $\alpha$ be any endomorphism of $B$ of rank $r$ with $r<m$. It follows from the lemma that $\alpha$ can be written as a product of endomorphisms (of $B$ ) of rank $m-1$. If we assume condition (1), then each factor in this product can be written as a product of idempotent endomorphisms. But by Lemma 2.6, each of these idempotents must have rank $m-1$, and hence $\alpha \in\left\langle E_{1}^{B}\right\rangle$. Thus (2) holds.

Our next aim is to show the equivalence of conditions (4) and (5).

Lemma 3.3. Let $A$ be a stable basis algebra and let $\varepsilon_{1}, \ldots, \varepsilon_{k}$ be idempotents of finite rank $r$ in End $A$. If $\varepsilon_{k} \varepsilon_{k-1} \cdots \varepsilon_{1}$ is regular of rank $r$, then for $i \in\{1, \ldots, k-1\}$ both $\varepsilon_{k} \cdots \varepsilon_{i}$ and $\varepsilon_{i+1} \varepsilon_{i}$ are regular of rank $r$.

Proof. From Lemma 2.8 we know that $\varepsilon_{k} \cdots \varepsilon_{i}$ and $\varepsilon_{i+1} \varepsilon_{i}$ have rank $r$. From Corollary 2.9 we have that

$$
\varepsilon_{k} \cdots \varepsilon_{1} \mathcal{R}^{*} \varepsilon_{k} \mathcal{R}^{*} \varepsilon_{k} \cdots \varepsilon_{i}
$$

so that by Lemma 2.1 we have that $\varepsilon_{k} \cdots \varepsilon_{i}$ is regular. Again using Corollary 2.9 we have that

$$
\varepsilon_{k} \cdots \varepsilon_{i} \mathcal{L}^{*} \varepsilon_{i} \mathcal{L}^{*} \varepsilon_{i+1} \varepsilon_{i}
$$

and now Lemma 2.1 tells us that $\varepsilon_{i+1} \varepsilon_{i}$ is regular. 
Lemma 3.4. Let $A$ be a stable basis algebra and let $\alpha_{1}, \ldots, \alpha_{k} \in$ End $A$ be regular of finite rank $r$ such that the product $\alpha_{k} \cdots \alpha_{1}$ is also regular of rank $r$. Then

$$
\text { Ker } \alpha_{k} \cdots \alpha_{1}=\operatorname{Ker} \alpha_{k}
$$

and

$$
\operatorname{Im} \alpha_{k} \cdots \alpha_{1}=\operatorname{Im} \alpha_{1} .
$$

Proof. From Corollary 2.9 we know that

$$
\alpha_{k} \mathcal{R}^{*} \alpha_{k} \cdots \alpha_{1} \mathcal{L}^{*} \alpha_{1}
$$

so that by Lemmas 2.2 and 2.3 we have that

$$
\operatorname{Ker} \alpha_{k}=\operatorname{Ker} \alpha_{k} \cdots \alpha_{1} \quad \text { and } \quad \operatorname{PC}\left(\operatorname{Im} \alpha_{k} \cdots \alpha_{1}\right)=\operatorname{PC}\left(\operatorname{Im} \alpha_{1}\right) .
$$

But $\alpha_{k} \cdots \alpha_{1}$ and $\alpha_{1}$ are regular, so that by Lemma 2.4 we have

$$
\operatorname{Im} \alpha_{k} \cdots \alpha_{1}=\operatorname{Im} \alpha_{1} .
$$

We are now in a position to prove that (4) implies (5).

Lemma 3.5. Let $B$ be a stable basis algebra and let $(\rho, C)$ be an endomorphism pair with $C$ of finite rank $r$. If $H_{(\rho, C)}^{*}$ contains a regular element which is a product of $k$ idempotents of rank $r$, then there are pure subalgebras $C_{1}, \ldots, C_{k}$ of $B$ and congruences $\rho_{1}, \ldots, \rho_{k}$ on $B$ with $C=C_{1}, \rho=\rho_{k}$ and such that for all $i, j$ with $1 \leqslant i \leqslant k$ and $1 \leqslant j \leqslant k-1,\left(\rho_{i}, C_{i}\right)$ and $\left(\rho_{j}, C_{j+1}\right)$ are idempotent endomorphism pairs.

Proof. Let $\alpha \in H_{(\rho, C)}^{*}$ be regular such that $\alpha=\varepsilon_{k} \cdots \varepsilon_{1}$ is a product of $k$ idempotents of rank $r$. For $1 \leqslant i \leqslant k$ put $C_{i}=\operatorname{Im} \varepsilon_{i}$ and $\rho_{i}=\operatorname{Ker} \varepsilon_{i}$, so that $\left(\rho_{i}, C_{i}\right)$ is an idempotent endomorphism pair. By Lemma 3.4 we have that

$$
C=\mathrm{PC}(\operatorname{Im} \alpha)=\operatorname{Im} \alpha=\operatorname{Im} \varepsilon_{k} \cdots \varepsilon_{1}=\operatorname{Im} \varepsilon_{1}=C_{1}
$$

and

$$
\rho_{k}=\operatorname{Ker} \varepsilon_{k}=\operatorname{Ker} \varepsilon_{k} \cdots \varepsilon_{1}=\operatorname{Ker} \alpha=\rho .
$$

From Lemma $3.3, \varepsilon_{j+1} \varepsilon_{j}$ is regular of rank $r$, for $1 \leqslant j \leqslant k-1$. Since

$$
\varepsilon_{j+1} \mathcal{R}^{*} \varepsilon_{j+1} \varepsilon_{j} \mathcal{L}^{*} \varepsilon_{j}
$$

and $\varepsilon_{j+1}, \varepsilon_{j+1} \varepsilon_{j}$ and $\varepsilon_{j}$ are regular, the comments at the beginning of $\S 2$ tell us that

$$
\varepsilon_{j+1} \mathcal{R} \varepsilon_{j+1} \varepsilon_{j} \mathcal{L} \varepsilon_{j} .
$$

By a classical result of semigroup theory (see, for example, [15, Proposition 2.3.7]), we have that $R_{\varepsilon_{j}} \cap L_{\varepsilon_{j+1}}$ contains an idempotent $\eta_{j}$. Certainly, then, $\eta_{j} \in R_{\varepsilon_{j}}^{*} \cap L_{\varepsilon_{j+1}}^{*}$. We have

$$
\operatorname{Ker} \eta_{j}=\operatorname{Ker} \varepsilon_{j}=\rho_{j},
$$


and as $\varepsilon_{j+1}$ is regular,

$$
\mathrm{PC}\left(\operatorname{Im} \eta_{j}\right)=\mathrm{PC}\left(\operatorname{Im} \varepsilon_{j+1}\right)=\operatorname{Im} \varepsilon_{j+1}=C_{j+1}
$$

so that $H_{\eta_{j}}^{*}=H_{\left(\rho_{j}, C_{j+1}\right)}^{*}$ and $\left(\rho_{j}, C_{j+1}\right)$ is an idempotent endomorphism pair.

As a consequence of the following lemma we have that (5) implies (4).

Lemma 3.6. Let $B$ be a stable basis algebra of rank $m$. Suppose that for any endomorphism pair $(\rho, C)$ with rank $C=m-1$ there are pure subalgebras $C_{1}, \ldots, C_{k}$ of $B$ and congruences $\rho_{1}, \ldots, \rho_{k}$ with $C=C_{1}, \rho=\rho_{k}$ and such that for all $i, j$ with $1 \leqslant i \leqslant k$ and $1 \leqslant j \leqslant k-1,\left(\rho_{i}, C_{i}\right)$ and $\left(\rho_{j}, C_{j+1}\right)$ are idempotent endomorphism pairs. Then every $\mathcal{H}^{*}$-class contained in $D_{m-1}^{*}$ contains a regular element that is a product of idempotents.

Proof. Let $(\rho, C)$ be an endomorphism pair with $\operatorname{rank} C=m-1$. Let $C_{1}, \ldots, C_{k}$ and $\rho_{1}, \ldots, \rho_{k}$ be chosen as in the statement of the lemma. For each $i \in\{1, \ldots, k\}$ let $\varepsilon_{i}=\varepsilon_{i}^{2} \in H_{\left(\rho_{i}, C_{i}\right)}^{*}$, and for each $j \in\{1, \ldots, k-1\}$ let $\eta_{j}=\eta_{j}^{2} \in H_{\left(\rho_{j}, C_{j+1}\right)}^{*}$. Consider the product $\varepsilon_{k} \cdots \varepsilon_{1}$.

Since

$$
\operatorname{Ker} \eta_{1}=\rho_{1}=\operatorname{Ker} \varepsilon_{1}
$$

and

$$
\operatorname{Im} \eta_{1}=C_{2}=\operatorname{Im} \varepsilon_{2}
$$

we have that

$$
\varepsilon_{2} \mathcal{L} \eta_{1} \mathcal{R} \varepsilon_{1}
$$

By Proposition 2.3.7 of [15],

$$
\varepsilon_{2} \mathcal{R} \varepsilon_{2} \varepsilon_{1} \mathcal{L} \varepsilon_{1}
$$

Suppose we have shown inductively that

$$
\varepsilon_{j} \mathcal{R} \varepsilon_{j} \cdots \varepsilon_{1} \mathcal{L} \varepsilon_{1}
$$

for $j \in\{1, \ldots, k-1\}$. By a now-familiar argument we have

$$
\varepsilon_{j+1} \mathcal{L} \eta_{j} \mathcal{R} \varepsilon_{j} \cdots \varepsilon_{1}
$$

so that again by Proposition 2.3.7 of [15],

$$
\varepsilon_{j+1} \mathcal{R} \varepsilon_{j+1} \cdots \varepsilon_{1} \mathcal{L} \varepsilon_{j} \cdots \varepsilon_{1} \mathcal{L} \varepsilon_{1} .
$$

By finite induction we have

$$
\varepsilon_{k} \mathcal{R} \varepsilon_{k} \cdots \varepsilon_{1} \mathcal{L} \varepsilon_{1}
$$

In particular, $\alpha=\varepsilon_{k} \cdots \varepsilon_{1}$ is regular,

$$
\operatorname{Im} \alpha=\operatorname{Im} \varepsilon_{1}=C_{1}=C
$$


and

$$
\operatorname{Ker} \alpha=\operatorname{Ker} \varepsilon_{k}=\rho_{k}=\rho,
$$

so that $\alpha \in H_{(\rho, B)}^{*}$. The result now follows from Proposition 2.10 .

Our next goal is to show that the first two conditions are linked to the last two by showing that conditions (2) and (4) are equivalent. One implication is easy: if (2) holds and $B$ is a pure subalgebra of $A$ with $\operatorname{rank} B \geqslant 2$, then, since $B$ is an stable basis algebra, Lemma 2.5 tells us that every $\mathcal{H}^{*}$-class of $\operatorname{End} B$ contains a regular element. Hence condition (4) holds.

We now proceed to show the reverse implication, which requires some care. The crux of the proof is in the following two lemmas.

Lemma 3.7. Let $A$ be a stable basis algebra with basis $\left\{x_{1}, x_{2}\right\}$ and define

$$
(\rho, C)=\left(\left\langle\left\langle\left(x_{1}, x_{2}\right)\right\rangle,\left\langle x_{2}\right\rangle\right) .\right.
$$

Then $(\rho, C)$ is an idempotent endomorphism pair and every element of $H_{(\rho, C)}^{*}$ is a product of idempotents.

Proof. That $(\rho, C)$ is an idempotent endomorphism pair follows from Lemma 2.11. Suppose that $\alpha \in H_{(\rho, C)}^{*}$. Then

$$
\operatorname{Im} \alpha=\left\langle x_{1} \alpha, x_{2} \alpha\right\rangle=\left\langle x_{2} \alpha\right\rangle
$$

and

$$
\operatorname{Im} \alpha \subseteq \operatorname{PC}(\operatorname{Im} \alpha)=\left\langle x_{2}\right\rangle .
$$

Define $\varepsilon_{1}, \varepsilon_{2} \in$ End $A$ by

$$
x_{1} \varepsilon_{1}=x_{2} \varepsilon_{2}=x_{1}
$$

and

$$
x_{1} \varepsilon_{2}=x_{2} \alpha, \quad x_{2} \varepsilon_{2}=x_{2} .
$$

Clearly, $\varepsilon_{1}^{2}=\varepsilon_{1}$ and $x_{2} \varepsilon_{2}=x_{2} \varepsilon_{2}^{2}$. Now $x_{2} \alpha=t\left(x_{2}\right)$ for some term $t$, which gives

$$
x_{1} \varepsilon_{2}^{2}=\left(x_{2} \alpha\right) \varepsilon_{2}=t\left(x_{2}\right) \varepsilon_{2}=t\left(x_{2} \varepsilon_{2}\right)=t\left(x_{2}\right)=x_{2} \alpha=x_{1} \varepsilon_{2}
$$

so that $\varepsilon_{2}^{2}=\varepsilon_{2}$. Further,

$$
x_{1} \varepsilon_{1} \varepsilon_{2}=x_{1} \varepsilon_{2}=x_{2} \alpha=x_{1} \alpha
$$

and

$$
x_{2} \varepsilon_{1} \varepsilon_{2}=x_{1} \varepsilon_{2}=x_{2} \alpha
$$

so that $\alpha=\varepsilon_{1} \varepsilon_{2}$. 
Lemma 3.8. Let $B$ be a stable basis algebra with basis $\left\{x_{1}, \ldots, x_{m}\right\}$ where $2 \leqslant m$ and define

$$
(\rho, C)=\left(\left\langle\left\langle\left(x_{1}, x_{2}\right)\right\rangle,\left\langle x_{2}, \ldots, x_{m}\right\rangle\right) .\right.
$$

Then $(\rho, C)$ is an idempotent endomorphism pair and, if $B$ satisfies condition (4), then every element of $H_{(\rho, C)}^{*}$ is a product of idempotents.

Proof. By Lemma 2.11, $(\rho, C)$ is certainly an idempotent endomorphism pair.

If $m=2$, the result is Lemma 3.7. Assume that $3 \leqslant m$ and that the lemma holds for all stable basis algebras of rank $m-1$.

By Lemmas 1.2 and 1.3, $C$ is a pure subalgebra of $B$, and is a stable basis algebra. Moreover, any pure subalgebra of $C$ is a pure subalgebra of $B$ and thus $C$ satisfies condition (4). Clearly, $C$ has rank $m-1$ and so the induction hypothesis applies. Hence there is an $\mathcal{H}^{*}$-class $H^{*}$ of End $C$ in $D_{m-2}^{*}$ containing an idempotent and such that every element of $H^{*}$ is a product of idempotents. Now, applying Proposition 2.13 to End $C$ we have that every element of $D_{m-2}^{*}$ can be written in the form $\gamma \beta \delta$ for some $\beta \in H^{*}$ and regular elements $\gamma, \delta$ in $D_{m-2}^{*}$. Now $\beta$ is a product of idempotents and, by condition (4), $\gamma$ and $\delta$ can be chosen to be products of idempotents. Thus every element of $D_{m-2}^{*}$ in End $C$ is a product of idempotents.

Let $\alpha \in H_{(\rho, C)}^{*}$ so that

$$
\operatorname{Im} \alpha=\left\langle x_{2} \alpha, \ldots, x_{m} \alpha\right\rangle,
$$

and as $\operatorname{rank} \alpha=m-1,\left\{x_{2} \alpha, \ldots, x_{m} \alpha\right\}$ is a basis for $\operatorname{Im} \alpha$. Now

$$
\operatorname{Im} \alpha \subseteq \operatorname{PC}(\operatorname{Im} \alpha)=\left\langle x_{2}, \ldots, x_{m}\right\rangle .
$$

Let $\beta \in$ End $C$ be defined by

$$
\begin{aligned}
& x_{2} \beta=x_{3} \alpha, \\
& x_{i} \beta=x_{i} \alpha, \quad 3 \leqslant i \leqslant m .
\end{aligned}
$$

Then

$$
\operatorname{Im} \beta=\left\langle x_{3} \alpha, \ldots, x_{m} \alpha\right\rangle
$$

so that $\operatorname{rank} \beta=m-2$, that is, $\beta \in D_{m-2}^{*}$. Hence there are idempotents $\varepsilon_{1}, \ldots, \varepsilon_{k}$ in End $C$ such that $\beta=\varepsilon_{1} \cdots \varepsilon_{k}$.

Define $\varepsilon_{i}^{\prime} \in$ End $B$ by

$$
\begin{aligned}
& x_{1} \varepsilon_{i}^{\prime}=x_{1}, \\
& x_{j} \varepsilon_{i}^{\prime}=x_{j} \varepsilon_{i}, \quad 2 \leqslant j \leqslant m .
\end{aligned}
$$

To see that each $\varepsilon_{i}^{\prime}$ is idempotent, notice that for $j \in\{2, \ldots, m\}$ we have $x_{j} \varepsilon_{i}=$ $t_{j}\left(x_{2}, \ldots, x_{m}\right)$ for some term $t_{j}$. Then

$$
\begin{aligned}
x_{j} \varepsilon_{i}^{\prime} \varepsilon_{i}^{\prime} & =\left(x_{j} \varepsilon_{i}\right) \varepsilon_{i}^{\prime}=t_{j}\left(x_{2}, \ldots, x_{m}\right) \varepsilon_{i}^{\prime}=t_{j}\left(x_{2} \varepsilon_{i}^{\prime}, \ldots, x_{m} \varepsilon_{i}^{\prime}\right) \\
& =t_{j}\left(x_{2} \varepsilon_{i}, \ldots, x_{m} \varepsilon_{i}\right)=t_{j}\left(x_{2}, \ldots, x_{m}\right) \varepsilon_{i}=x_{j} \varepsilon_{i} \varepsilon_{i}=x_{j} \varepsilon_{i}=x_{j} \varepsilon_{i}^{\prime} .
\end{aligned}
$$


We define two further idempotents in End $B, \varphi$ and $\eta$, by putting

$$
\begin{aligned}
& x_{1} \varphi=x_{2} \varphi=x_{1}, \\
& x_{i} \varphi=x_{i}, \quad 3 \leqslant i \leqslant m, \\
& x_{1} \eta=x_{1} \alpha, \\
& x_{i} \eta=x_{i}, \quad 2 \leqslant i \leqslant m .
\end{aligned}
$$

Clearly, $\varphi$ is idempotent and since $\operatorname{Im} \eta \subseteq C$ and $\eta$ is the identity on $C, \eta$ is also idempotent. For $i \in\{1,2\}$ we have

$$
x_{i} \varphi \varepsilon_{1}^{\prime} \cdots \varepsilon_{k}^{\prime} \eta=x_{1} \eta=x_{i} \alpha .
$$

Let $j \in\{3, \ldots, m\}$. Then

$$
x_{j} \varphi \varepsilon_{1}^{\prime} \cdots \varepsilon_{k}^{\prime} \eta=x_{j} \varepsilon_{1}^{\prime} \cdots \varepsilon_{k}^{\prime} \eta .
$$

But $x_{j} \in C$ and each $\varepsilon_{i}^{\prime}$ maps $C$ into $C$; moreover, the restriction of $\varepsilon_{i}^{\prime}$ to $C$ is $\varepsilon_{i}$, and it follows that

$$
x_{j} \varphi \varepsilon_{1}^{\prime} \cdots \varepsilon_{k}^{\prime} \eta=x_{j} \varepsilon_{1} \cdots \varepsilon_{k} \eta=x_{j} \beta \eta=x_{j} \alpha \eta=x_{j} \alpha
$$

since $\eta$ is the identity on $C$. Hence $\alpha=\varphi \varepsilon_{1}^{\prime} \cdots \varepsilon_{k}^{\prime} \eta$, as required.

If $B$ is a stable basis algebra of finite rank $m \geqslant 2$ which satisfies condition (4), then it follows from Lemma 3.8 and Proposition 2.13 (as in the above proof) that every element of $D_{m-1}^{*}$ is a product of idempotents (of rank $m-1$ ). It now follows from Lemma 3.2 that $\left\langle E_{1}^{B}\right\rangle=S_{m-1}^{B}$. We can now conclude that if $A$ is a stable basis algebra which satisfies condition (4), then condition (2) holds for $A$.

Finally, we show that (3) is a consequence of the remaining conditions. Suppose that condition (2) holds, let $B$ be a pure subalgebra of $A$ with rank $m \geqslant 2$, and consider an $\mathcal{H}^{*}$-class $H^{*}$ of End $B$ consisting of endomorphisms of rank $r$, where $1 \leqslant r \leqslant m-1$. By Lemma 2.5, $H^{*}$ contains a regular element, say $\alpha$. By condition (2), we know that $\alpha=\varepsilon_{1} \cdots \varepsilon_{k}$ for some idempotents $\varepsilon_{i}$ of rank $m-1$. We now call upon Lemma 1 of [13] to write $\alpha$ as a product $\nu_{1} \cdots \nu_{k}$ of idempotents $\mathcal{D}$-related to $\alpha$. But $\mathcal{D} \subseteq \mathcal{D}^{*}$, so the idempotents $\nu_{i}$ have rank $r$ and are thus in $E_{m-r}^{B}$.

In view of Proposition 2.13, to complete the proof that (3) holds it is enough to show that every element of $H_{(\rho, C)}^{*}$ is a product of idempotents of rank $r$, where

$$
(\rho, C)=\left(\left\langle\left\langle\left(x_{1}, y_{1}\right), \ldots,\left(x_{1}, y_{m-r}\right)\right\rangle,\left\langle x_{1}, \ldots, x_{r}\right\rangle\right)\right.
$$

and $\left\{x_{1}, \ldots, x_{r}, y_{1}, \ldots, y_{m-r}\right\}$ is a basis for $B$. By Lemma 2.11 we know that $(\rho, C)$ is an idempotent endomorphism pair for $B$. Let $\beta \in H_{(\rho, C)}^{*}$. Then

$$
\operatorname{Im} \beta=\left\langle x_{1} \beta, \ldots, x_{r} \beta, y_{1} \beta, \ldots, y_{m-r} \beta\right\rangle=\left\langle x_{1} \beta, \ldots, x_{r} \beta\right\rangle .
$$

Put $D=\left\langle x_{1}, \ldots, x_{r}, y_{1}\right\rangle$. Then $\operatorname{Im} \beta \subseteq D$ and $D$ is a pure subalgebra of $A$. Let $\gamma$ be the restriction of $\beta$ to $D$, so that $\gamma \in \operatorname{End} D$. Now $\operatorname{Im} \gamma=\operatorname{Im} \beta$ so that $\operatorname{rank} \gamma=r$. We know that $\operatorname{rank} D=r+1$ so, by (2), there are idempotents $\varepsilon_{i} \in$ End $D$ such that 
$\gamma=\varepsilon_{1} \cdots \varepsilon_{k}$ and $\operatorname{rank} \varepsilon_{i}=r$, for $i \in\{1, \ldots, k\}$. Extend each $\varepsilon_{i}$ to an endomorphism $\varepsilon_{i}^{\prime}$ of $B$ by putting

$$
\begin{array}{ll}
x_{j} \varepsilon_{i}^{\prime}=x_{j} \varepsilon_{i}, & \text { for } 1 \leqslant j \leqslant r, \\
y_{j} \varepsilon_{i}^{\prime}=y_{1} \varepsilon_{i}, & \text { for } 1 \leqslant j \leqslant m-r .
\end{array}
$$

Now, for any $i \in\{1, \ldots, k\}, \operatorname{Im} \varepsilon_{i}^{\prime}=\operatorname{Im} \varepsilon_{i}$ and, as $\varepsilon_{i}$ is the restriction of $\varepsilon_{i}^{\prime}$ to $D$, it follows that each $\varepsilon_{i}^{\prime}$ is idempotent and $\operatorname{rank} \varepsilon_{i}^{\prime}=\operatorname{rank} \varepsilon_{i}=r$. Further, for $j \in\{1, \ldots, r\}$ we have

$$
x_{j} \varepsilon_{1}^{\prime} \cdots \varepsilon_{k}^{\prime}=x_{j} \varepsilon_{1} \cdots \varepsilon_{k}=x_{j} \gamma=x_{j} \beta
$$

and, for $\ell \in\{1, \ldots, m-r\}$, we have

$$
y_{\ell} \varepsilon_{1}^{\prime} \cdots \varepsilon_{k}^{\prime}=y_{1} \varepsilon_{1} \varepsilon_{2}^{\prime} \cdots \varepsilon_{k}^{\prime}=y_{1} \varepsilon_{1} \varepsilon_{2} \cdots \varepsilon_{k}=y_{1} \gamma=y_{1} \beta=y_{\ell} \beta .
$$

It follows that $\beta=\varepsilon_{1}^{\prime} \cdots \varepsilon_{k}^{\prime}$ and, consequently, (3) holds.

\section{Examples}

In this section, we illustrate Theorem 3.1 and relate it to known results by considering some specific examples of stable basis algebras.

\subsection{Independence algebras}

An independence algebra $A$ of finite rank $n$ is certainly a stable basis algebra. It is known [10] (see also [1]) that every endomorphism of $A$ of rank strictly less than $n$ is a product of idempotent endomorphisms. We can also obtain this result by using Theorem 3.1 and a preliminary result of [10]. First, we recall that in this case End $A$ is regular, so that $\mathcal{H}^{*}=\mathcal{H}$, etc. Now, by Corollary 2.4 of [10], every $\mathcal{H}$-class contained in $D_{n-1}$ contains an element which is a product of (at most three) idempotents. Since this is true for all $n$, it follows that condition (4) of Theorem 3.1 holds.

\subsection{Modules}

As mentioned in the introduction, finitely generated free modules over Bezout domains are stable basis algebras, and so we can specialize Theorem 3.1 to this case. For an endomorphism of a module, the kernel is a submodule, and specializing the notion of endomorphism pair leads to that of a weakly complementary pair of finitely generated pure submodules. Recall that a submodule $N$ of a torsion-free $R$-module over an integral domain $R$ is pure if whenever $r m \in N$ for some $r \in R, r \neq 0, m \in M$ we have $m \in N$. Let $R$ be a Bezout domain and let $F$ be a finitely generated free module over $R$. Let $A, B$ be finitely generated pure submodules of $F$. The pair $(A, B)$ is complementary if $F=A+B$ and $A \cap B=0$ (this corresponds to an idempotent endomorphism pair); it is weakly complementary if for some positive integer $k$ there are finitely generated pure submodules $C_{1}, \ldots, C_{k}, D_{1}, \ldots, D_{k}$ with $A=C_{1}, B=D_{k}$ and such that the pairs $\left(C_{i}, D_{i}\right)$ and $\left(C_{j+1}, D_{j}\right)$ are complementary for $i=1, \ldots, k$ and $j=1, \ldots, k-1$. Then 
the specialization of Theorem 3.1 gives part of the main theorem of [18], which also provides two further conditions equivalent to the others:

(6) every invertible $2 \times 2$ matrix over $R$ is a product of elementary matrices;

(7) every invertible matrix over $R$ is a product of elementary matrices.

In general, integral domains which satisfy (6) are called $\mathrm{GE}_{2}$-rings in [3], and those which satisfy (7) are called GE-rings. Here, 'GE' stands for generalized Euclidean, and as Ruitenberg [18] points out, it is well known that a matrix over a Euclidean domain can be reduced to a diagonal matrix by elementary row and column operations so that condition (7) holds for such a domain. Euclidean domains are certainly Bezout domains, and so, for all $n \geqslant 2$, every $n \times n$ matrix over a Euclidean domain of rank at most $n-1$ is a product of idempotent matrices. This result was also proved by Laffey [16], who reduced the problem to the case of $2 \times 2$ matrices. Using this reduction (or Ruitenberg's result), an alternative approach would be to verify condition (5) of Theorem 3.1 for $n=2$, as described below.

Let $R$ be a (commutative) principal ideal domain. Clearly, the submodule $\langle(a, b)\rangle$ of $R^{2}$ is pure if and only if $a, b$ are coprime, and in this case we say that the pair $(a, b)$ is pure. Also, it is not difficult to see that the pure submodules $\langle(a, b)\rangle,\langle(c, d)\rangle$ form a complementary pair if and only if the matrix

$$
\left(\begin{array}{ll}
a & c \\
b & d
\end{array}\right)
$$

is invertible. Let $\left(a_{1}, b_{1}\right),\left(a_{2}, b_{2}\right)$ be pure pairs in $R^{2}$. We say that there is a link from $\left(a_{1}, b_{1}\right)$ to $\left(a_{2}, b_{2}\right)$ if, for some positive integer $k$, there are pairs $\left(c_{i}, d_{i}\right),\left(e_{i}, f_{i}\right)$ for $i=$ $1, \ldots, k$ such that $\left(a_{1}, b_{1}\right)=\left(c_{1}, d_{1}\right),\left(a_{2}, b_{2}\right)=\left(e_{k}, f_{k}\right)$ and the matrices

$$
\left(\begin{array}{cc}
c_{i} & e_{i} \\
d_{i} & f_{i}
\end{array}\right) \text { and }\left(\begin{array}{cc}
c_{j+1} & e_{j} \\
d_{j+1} & f_{j}
\end{array}\right)
$$

are invertible for $i=1, \ldots, k$ and $j=1, \ldots, k-1$.

We note that condition (5) for $R^{2}$ becomes

$\left(5^{\prime}\right)$ for any two pure pairs $\left(a_{1}, b_{1}\right),\left(a_{2}, b_{2}\right)$ in $R^{2}$, there is a link from $\left(a_{1}, b_{1}\right)$ to $\left(a_{2}, b_{2}\right)$.

Suppose that there is a link as described above from $\left(a_{1}, b_{1}\right)$ to $\left(a_{2}, b_{2}\right)$. Then by reversing the sequence of matrices and interchanging the columns in each matrix we see that there is a link from $\left(a_{2}, b_{2}\right)$ to $\left(a_{1}, b_{1}\right)$.

Now suppose that there is a link from $\left(a_{1}, b_{1}\right)$ to $\left(a_{2}, b_{2}\right)$ and a link from $\left(a_{2}, b_{2}\right)$ to $\left(a_{3}, b_{3}\right)$. Say the pairs $\left(c_{i}, d_{i}\right)$ and $\left(e_{i}, f_{i}\right)$ for $i=1, \ldots, k$ give a link from $\left(a_{1}, b_{1}\right)$ to $\left(a_{2}, b_{2}\right)$, and the pairs $\left(r_{j}, s_{j}\right)$ and $\left(t_{j}, u_{j}\right)$ for $j=1, \ldots, h$ give a link from $\left(a_{2}, b_{2}\right)$ to $\left(a_{3}, b_{3}\right)$. Then the matrix

$$
\left(\begin{array}{ll}
c_{k} & e_{k} \\
d_{k} & f_{k}
\end{array}\right)
$$


is invertible and so there are elements $x, y$ in $R$ such that

$$
x c_{k}+y d_{k}=1=x e_{k}+y f_{k} .
$$

It is now easy to see that the sequences of pairs

$$
\left(c_{1}, d_{1}\right), \ldots,\left(c_{k}, d_{k}\right),(y,-x),\left(r_{1}, s_{1}\right), \ldots,\left(r_{h}, s_{h}\right)
$$

and

$$
\left(e_{1}, f_{1}\right), \ldots,\left(e_{k}, f_{k}\right),\left(c_{k}, d_{k}\right),\left(t_{1}, u_{1}\right), \ldots,\left(t_{h}, u_{h}\right)
$$

give a link from $\left(a_{1}, b_{1}\right)$ to $\left(a_{3}, b_{3}\right)$.

Finally, observe that if the pairs $\left(c_{i}, d_{i}\right)$ and $\left(e_{i}, f_{i}\right)$ for $i=1, \ldots, k$ give a link from $\left(a_{1}, b_{1}\right)$ to $(u, 0)$, where $u$ is a unit, then replacing $(u, 0)$ (that is, $\left.\left(e_{k}, f_{k}\right)\right)$ by $(1,0)$ gives a link from $\left(a_{1}, b_{1}\right)$ to $(1,0)$.

These observations together show that condition $\left(5^{\prime}\right)$ is equivalent to

$\left(5^{\prime \prime}\right)$ for any pure pair $(a, b)$ in $R^{2}$, there is a link from $(a, b)$ to $(u, 0)$ for some unit $u$.

Now suppose that $R$ is a Euclidean domain with valuation $\delta$, and let $(a, b)$ be a pure pair in $R^{2}$. If $a=0$, then $b$ must be a unit, and so taking $\left(e_{1}, f_{1}\right)=(1,0)$ gives a link from $(a, b)$ to $(1,0)$. If $a$ is a unit, then taking $\left(e_{1}, f_{1}\right)=(0,1)$ gives a link from $(a, b)$ to $(0,1)$, and hence this can be extended to give a link to $(1,0)$.

Now let $a$ be a non-zero non-unit so that $\delta(a)>\delta(1)$ and suppose inductively that for all pure $(c, d)$ with $\delta(c)<\delta(a)$, there is a link from $(c, d)$ to $(u, 0)$ for some unit $u$. It now suffices to show that there are elements $c, d, e, f$ in $R$ with $c=0$ or $\delta(c)<\delta(a)$ such that the matrices

$$
\left(\begin{array}{ll}
a & e \\
b & f
\end{array}\right) \text { and }\left(\begin{array}{ll}
c & e \\
d & f
\end{array}\right)
$$

are invertible. Since $a, b$ are coprime, there are elements $x, y$ in $R$ with $a x+b y=1$. Since $a$ is not a unit, it cannot be a factor of $y$ and so $y=q a+r$ for some $q, r$ with $\delta(r)<\delta(a)$. Put $e=-r$ and $f=x+b q$ so that $a f-b e=1$. If $e$ is a unit, put $c=0$, $d=1$. Otherwise, the same argument tells us that $e d-f c=1$ for some $d, c \in R$ with $c \neq 0$ and $\delta(c)<\delta(r)=\delta(e)<\delta(a)$.

Thus the required elements exist.

Laffey [16] also raised the question of whether, for all $n$, every $n \times n$ matrix of rank at most $n-1$ over an arbitrary principal ideal domain $R$ can be written as a product of idempotents. The answer is no: in view of Ruitenberg's result that (6) and (7) are equivalent to this property, the question is equivalent to asking if all principal ideal domains are $\mathrm{GE}_{2}$-rings. In $[\mathbf{3}]$, Cohn shows that, among other examples, the ring of integers in $\mathbb{Q}(\sqrt{-19})$ is not a $\mathrm{GE}_{2}$-ring.

\subsection{Acts}

Our third canonical example of a basis algebra is a free left $T$-set over a cancellative left Ore domain. We remark that if $T$ is a monoid in which every finitely generated left 
ideal is principal, then $T$ is certainly left Ore. If $s, t \in T$, then $T s \cup T t$ is principal, whence $T s \cup T t=T t$ or $T s \cup T t=T s$. It follows that $s=r t$ or $t=r s$ for some $r \in T$.

Lemma 4.1. Let $A$ be a free left $T$-act on a set $X$ with $|X|=n$, where $T$ is a cancellative monoid in which every finitely generated left ideal is principal. Then $A$ is a stable basis algebra.

Proof. We call upon the results of [8]. Since every finitely generated left ideal of $T$ is principal, it follows that $T$ is left Ore. Certainly $A$ is faithful as a left $T$-act, whence in the notation of $[\mathbf{8}], T \cong T_{1}^{*}=T_{1}$. From Corollary 5.6 and Lemma 7.1 of [8], together with the fact that $A$ is relatively free with basis $X$, we have that $A$ is a basis algebra. Now Proposition 7.5 of $[\mathbf{8}]$ tells us that $A$ is stable.

It is easy to see that the pure subalgebras of $A$ all have the form

$$
B=\bigcup_{y \in Y} T y,
$$

where $Y \subseteq X$ and any basis of $B$ is of the form

$$
\left\{u_{y} y: y \in Y\right\}
$$

where each $u_{y}$ is a unit of $T$.

Theorem 4.2. Let $A$ be a free left $T$-act on a set $X$ with $|X|=n \geqslant 2$, where $T$ is a cancellative monoid in which every finitely generated left ideal is principal. Then every endomorphism of $A$ of rank less than $n$ is a product of idempotent endomorphisms.

Proof. Let $B$ be a free $T$-act on a set $Y$ where $2 \leqslant|Y|=m$. We show that every regular element of End $B$ of rank $m-1$ is a product of idempotents. The required result then follows from Proposition 2.5 and Theorem 3.1.

Let $\alpha \in D_{m-1}^{*}$ be regular. Then

$$
\operatorname{Im} \alpha=\mathrm{PC}(\operatorname{Im} \alpha)=C,
$$

where $\operatorname{rank} C=m-1$. Put $Y=\left\{y_{1}, \ldots, y_{m}\right\}$ and without loss of generality suppose that

$$
C=T y_{2} \cup \cdots \cup T y_{m}
$$

Now

$$
\operatorname{Im} \alpha=\left\langle y_{1} \alpha, \ldots, y_{m} \alpha\right\rangle=\left\langle y_{2}, \ldots, y_{m}\right\rangle
$$

and it follows that for some $i<j$ we must have

$$
\left\langle y_{i} \alpha, y_{j} \alpha\right\rangle=\left\langle y_{a(i)}\right\rangle
$$

and, for $k \in\{1, \ldots, m\} \backslash\{i, j\}$,

$$
\left\langle y_{k} \alpha\right\rangle=\left\langle y_{a(k)}\right\rangle,
$$


where

$$
a:\{1, \ldots, m\} \backslash\{i, j\} \rightarrow\{2, \ldots, m\} \backslash\{a(i)\}
$$

is a bijection.

Again without loss of generality we may assume that

$$
y_{i} \alpha=r y_{a(i)}, \quad y_{j} \alpha=s y_{a(i)} \quad \text { and } \quad s=t r
$$

for some $r, s, t \in T$. Thus

$$
y_{j} \alpha=s y_{a(i)}=\operatorname{tr} y_{a(i)}=t y_{i} \alpha,
$$

and further,

$$
\left\langle y_{i} \alpha\right\rangle=\left\langle y_{a(i)}\right\rangle .
$$

From the proof of Lemma 4.1 we deduce that $r \in G$, where $G$ is the group of units of $T$, and, for $k \in\{1, \ldots, n\} \backslash\{i, j\}$,

where each $u_{k} \in G$.

$$
y_{k} \alpha=u_{k} y_{a(k)},
$$

Define $\beta \in$ End $B$ by

so that $\beta=\beta^{2}$.

$$
y_{\ell} \beta= \begin{cases}s r^{-1} y_{i} & \text { if } \ell=j \\ y_{\ell} & \text { otherwise }\end{cases}
$$

Now consider the $G$-set $D=G y_{1} \cup \cdots \cup G y_{m}$. From [11] we have that $D$ is an independence algebra. Define $\gamma \in$ End $D$ by

$$
y_{k} \gamma= \begin{cases}r y_{a(i)} & \text { if } k=i, \\ y_{a(i)} & \text { if } k=j, \\ u_{k} y_{a(k)} & \text { otherwise }\end{cases}
$$

Clearly, rank $\gamma=m-1$. From Theorem 2.1 of [10] we know that End $D$ has our desired property, namely, $\gamma=\varepsilon_{1} \cdots \varepsilon_{p}$ for some idempotents $\varepsilon_{i} \in$ End $D$. For each $i \in\{1, \ldots, p\}$ we define $\varepsilon_{i}^{\prime} \in \operatorname{End} B$ by

$$
y \varepsilon_{i}^{\prime}=y \varepsilon_{i} \quad \text { for all } y \in Y .
$$

Since $\varepsilon_{i}^{\prime}$ extends $\varepsilon_{i}$, it is clear that $\varepsilon_{i}^{\prime}$ is idempotent for all $i \in\{1, \ldots, p\}$. We claim that $\alpha=\beta \varepsilon_{1}^{\prime} \cdots \varepsilon_{p}^{\prime}$.

Using the fact that each $\varepsilon_{i}$ maps $D$ to $D$, and is the restriction to $D$ of $\varepsilon_{i}^{\prime}$, we have, for $k \in\{1, \ldots, m\} \backslash\{i, j\}$,

$$
y_{k} \beta \varepsilon_{1}^{\prime} \cdots \varepsilon_{p}^{\prime}=y_{k} \varepsilon_{1}^{\prime} \cdots \varepsilon_{p}^{\prime}=y_{k} \varepsilon_{1} \cdots \varepsilon_{p}=y_{k} \gamma=u_{k} y_{a(k)}=y_{k} \alpha
$$

and, similarly,

Finally,

$$
y_{i} \beta \varepsilon_{1}^{\prime} \cdots \varepsilon_{p}^{\prime}=y_{i} \varepsilon_{1}^{\prime} \cdots \varepsilon_{p}^{\prime}=y_{i} \gamma=r y_{a(i)}=y_{i} \alpha
$$

$$
\begin{aligned}
y_{j} \beta \varepsilon_{1}^{\prime} \cdots \varepsilon_{p}^{\prime} & =\left(s r^{-1} y_{i}\right) \varepsilon_{1}^{\prime} \cdots \varepsilon_{p}^{\prime}=\left(s r^{-1}\right)\left(y_{i} \varepsilon_{1}^{\prime} \cdots \varepsilon_{p}^{\prime}\right) \\
& =\left(s r^{-1}\right)\left(y_{i} \gamma\right)=\left(s r^{-1}\right)\left(r y_{a(i)}\right)=s y_{a(i)}=y_{j} \alpha .
\end{aligned}
$$

It follows that $\alpha=\beta \varepsilon_{1}^{\prime} \cdots \varepsilon_{p}^{\prime}$, completing the proof as required. 


\section{References}

1. J. ARAúJo, Idempotent generated endomorphisms of an independence algebra, Semigroup Forum 67 (2003), 464-467.

2. P. M. Cohn, Universal algebra (Harper \& Row, New York, 1965).

3. P. M. Cohn, On the structure of the $\mathbf{G L}_{2}$ of a ring, Publ. Math. IHES 30 (1966), 5-53.

4. R. J. H. DAwlings, The semigroup of singular endomorphisms of a finite dimensional vector space, in Semigroups: Proc. Conf., Monash Univ., Clayton, Victoria, 1979 (ed. T. E. Hall, P. R. Jones and G. B. Preston), pp. 121-131 (Academic Press, 1980).

5. J. A. ERDős, On products of idempotent matrices, Glasgow Math. J. 8 (1967), 118-122.

6. J. Fountain, Abundant semigroups, Proc. Lond. Math. Soc. 44 (1982), 103-129.

7. J. Fountain, Products of idempotent integer matrices, Math. Proc. Camb. Phil. Soc. 110 (1991), 431-441.

8. J. Fountain And V. Gould, Relatively free algebras with weak exchange properties, $J$. Austral. Math. Soc. 75 (2003), 355-384 (see also www-users.york.ac.uk/ varg1).

9. J. Fountain AND V. Gould, Endomorphisms of relatively free algebras with weak exchange properties, Alg. Univers. 51 (2004), 257-285 (see also www-users.york.ac.uk/ $\sim \operatorname{varg} 1$ ).

10. J. Fountain And A. LeWin, Products of idempotent endomorphisms of an independence algebra of finite rank, Proc. Edinb. Math. Soc. 35 (1992), 493-500.

11. V. Gould, Independence algebras, Alg. Univers. 33 (1995), 294-318.

12. G. Grätzer, Universal algebra (Van Nostrand, Princeton, NJ, 1968).

13. T. E. HALL, On regular semigroups, J. Alg. 24 (1973), 1-24.

14. J. M. HowiE, The subsemigroup generated by the idempotents of a full transformation semigroup, J. Lond. Math. Soc. 41 (1966), 707-716.

15. J. M. HowIE, Fundamentals of semigroup theory (Oxford University Press, 1995).

16. T. LAFFEY, Products of idempotent matrices, Linear Multilinear Alg. 14 (1983), 309-314.

17. R. N. McKenzie, G. F. MCNulty And W. T. TAYlor, Algebra, lattices, varieties (Wadsworth, Monterey, CA, 1983).

18. W. Ruitenberg, Products of idempotent matrices over Hermite domains, Semigroup Forum 46 (1993), 371-378. 